\title{
Novas combinações em Angostura Roemer \& Schultes (Rutaceae)
}

\author{
Byron W. P. de Albuquerque \\ Instituto Nocional de Pesquisas \\ do Amazônia, Manous
}

Com base no fato de que o trabaiho de Brown (In: A. Flinders, Voy. Terra Australis 2: App. 3, 545, 1814) não podia ser aceito como a publicação válida para o nome genérico, Elias (Taxon 14 (4): 573-575, 1970) propôs Angostura Roemer \& Schultes (Systema Vegetalibium 2: 188. 1819) para substituir Cusparia
Humboldt, pertencente a tribo Cusparieae. Brown não incluiu uma única palavra de descrição e não citou qualquer descrição anteriormente publicada. Apenas referiu se a Cusparia à Ordem Diosmeae. Desse modo, tornou-se necessário localizar a primeira publicação válida para o nome genérico. Elias encontrou um 
nome aceitável para a correção, o gênero $A n$ gostura, descrito por Roemer \& Schultes com uma só espécie, $A$. cuspare, que passou a ter prioridade.

Até o momento, com exceção de Cowan \& Smith (FI. Ilustr. Catar. 1 pt. fasc. Ruta, p. 51. 1973) não foram mais publicadas outras com. binações em Angostura. Assim, as transferências novas necessárias para este gênero são:

Angostura acuminata (Pilger) B.W.P. de Albuquerque, comb. nov.

Cusparia acuminata Pilger, Verhandl. Bot. Brandenburg 47: 154. 1905.

Angostura candolleana (St.-Hilaire) B.W.P. de de Albuquerque, comb. nov.

Galipea candollesna St.-Hilaire, Bull. Soc. Philom. 132. 1823 e Hist. PI. Rem.

Brés. et Parag. 1:135. 1825.

Cusparia candolleana (St.-Hilaire) Engler, in Martius, Flora Brasiliensis

12 (2): 119. 1874.

Angostura cuneifolia (St.-Hilaire) B.W.P. de Albuquerque, comb. nov.

Galipea cuneifolia St.-Hilaire ex-DC. Prodr. 1: 732.1824.

Cusparia cuneifolia (St.-Hilaire) Engler, in Martius, Flora Brasiliensis 12 (2): 116. 1874.

Angostura elegans (St.-Hilaire) B.W.P: de Albuquerque, comb. nov.

Galipea elegans St.-Hilaire, FI. Bras. mer. 1: 90, 1825.

Cusparia elegans (St.-Hilaire) Engler, in Martius, Flora Brasiliensis 12 (2): 121, tab. 25, fig. 2. 1874.

Angostura engleriana (Taubert) B.W.P. de Albuquerque, comb. nov.

Cusparia engleriana Taubert, Bot. Jahrb. 15, Beibl. n. 34, 4. 1892.

Angostura fanshawei (Sandwith) B.W.P. de Albuquerque, comb. nov.
Cusparia fanshawei Sandwith, Kew Bull. 1948: 306. 1948.

Angostura fontanesiana (St.-Hilaire) B.W.P. de Albuquerque, comb. nov.

Galipea fontanesiana St.-Hilaire, Bull. Soc. Philom. 132. 1823 e Hist. PI. Rem.

Brés. et Parag. 1: 134, t. 14 B. 1825.

Cusparia fontanesiana (St.-Hilaire) Engler, in Martius, Flora Brasiliensis 12 (2): 122. 1874.

Angostura gaudichaudiana (St.-Hilaire) B.W.P. de Albuquerque, comb. nov.

Galipea gaudichaudiana St.-Hilaire, FI. Bras. mer. 1: 89. 1825.

Cusparia gaudichaudiana (St.-Hilaire) Enyier, in Martius, Flora Erasiliensis 12 (2): 116. 1874.

Angostura glazioviana (Taubert) B.W.P. de Albuquerque, comb. nov.

Cusparia glazioviana Taubert, Bot. Jahrb. 15, Beibl. n. 34, 5. 1892.

Angostura grandiflora (Engler) B.W.P. de Albuquerque, comb. nov.

Cusparia grandiflora Engler, in Martius, Flora Brasiliensis 12 (2): 112. 1874.

Angostura heterophylla (St.-Hilaire) B.W.P. de Albuquerque, comb. nov.

Galipea heterophylla St.-Hilaire, Bull. Soc. Philom. 132. 1823 e Hist. PI. Rem.

Brés. et Parag. 1: 131, t. 12. 1825.

Cusparia heterophylla (St.-Hilaire) Engler, in Martius, Flora Brasiliensis 12 (2): 114. 1874.

Angostura larensis (Tamayo \& Croizat) B.W.P. de Albuquerque, comb. nov.

Raputia larensis Tamayo \& Croizat, Lilloa 17: 223-226. 1949.

Cusparia larensis (Tamayo \& Croizat) Emmerich, Bradeae 2 (26): 117-180. 1977. 
Angostura macrocarpa (Engler) B.W.P. de Albuquerque, comb. nov.

Cusparia macrocarpa Engler, in Martius, Fiora Brasiliensis 12 (2): 121. 1874.

Angostura macrophylla (Mikan) B.W.P. de AIbuquerque, comb. nov.

Conchocarpus macrophyllus Mikan, Delect. FI. \& Faun. Brasil. fasc. 1.

t. 2. 1820 .

Galipea macrophylla St.-Hilaire, Mém. Mus. Paris 10: 282. 1823 e Hist. PI. Rem.

Brés. et Parag. 1: 132. 1825.

Cusparia macrophylla (Mikan) Engler, in Martius, Flora Brasiliensis 12 (2): 115, tab. 23, fig. 2. 1874.

Angostura magdalenensis (Cuatrecasas) B.W.P. de Albuquerque, comb. nov.

Cusparia magdalenensis Cuatrecasas, Rev. Acad. Colomb. Cienc. 8 (32): 465. 1952.

Angostura martiana (St.-Hilaire) B.W.P. de Albuquerque, comb. nov.

Galipea martiana St.-Hilaire, Fl. Bras. mer. 1: $90,1825$.

Cusparia martiana (St.-Hilaire) Engler, in Martius, Flora Brasiliensis 12

(2): 119. 1874.

Angostura nodosa (Engler) B.W.P. de Albuquerque, comb. nov.

Cusparia (?) nodosa Engler, in Martius, Flora Brasiliensis 12 (2): 123. 1874.

Angostura obovata (Nees \& Martius) B.W.P. de Albuquerque, comb. nov.

Diglottis obovata Nees \& Martius, Nov. Act. Nat. Cur. 11: 170, tab. 19, fig. F et tab. 25. 1823.

Cusparia obovata (Nees \& Martius) Engler, in Martius, Flora Brasiliensis 12

(2): $118,1874$.
Angostura ovata (St.-Hilaire \& Tulasne) B.W.P. de Albuquerque, comb. nov.

Galipea ovata St.-Hilaire \& Tulasne, Ann. Sc. Nat. 2, sér. 17, 139. 1842.

Cusparia ovata (St.-Hilaire \& Tulasne) Engler, in Martius, Flora Brasiliensis 12 (2): 117. 1874.

Angostura paniculata (Engler) B.W.P. de Albuquerque, comb. nov.

Cusparia paniculata Engler, in Martius, Flora Brasiliensis 12 (2): 120, tab. 24 , fig. 2. 1874 .

Angostura pentagyna (St.-Hilaire) B.W.P. de Albuquerque, comb. nov.

Galipea pentagyna St.-Hilaire, Mém. Mus. Paris 10: 281. 1823 e Hist. PI. Rem.

Brés. et Parag. 1: 131, tab. $11 \mathrm{~A}$. 1825.

Cusparia pentagyna (St.-Hilaire) Engler, in Martius, Flora Brasiliensis 12 (2): 122. 1874 .

Angostura pentandrá (St.-Hilaire) B.W.P. de Albuquerque, comb. nov.

Galipea pentandra St.-Hilaire, Bull. Soc. Philom. 132. 1823 e Hist. PI. Rem. Brés. et Parag. 1: 134. 1825.

Cusparia pentandra (St.-Hilaire) Engler, in Martius, Flora Brasiliensis 12

(2): 120.1874

Angostura pilocarpoidia (Rusby) B.W.P. de Albuquerque, comb. nov.

Cusparia pilocarpoidia Rusby, Bull. N.Y. Bot. Gard. 8: 98. 1912.

Angostura ramiflora (Engler) B.W.P. de Albuquerque, comb. nov.

Cusparia ramiflora Engler, in Martius, Flora Brasiliensis 12 (2): 111. 1874.

Galipea ramiflora Spruce, I. c., nom. nud. Angostura silvestris (Nees \& Martius) B.W.P. de Albuquerque, comb. nov. 
Lasiostemon silvestre Nees \& Martius, Nov. Act. Cur. 11: 171, tab. 19. 1823.

Cusparia silvestris (Nees \& Martius) Engler, in Martius, Flora Brasiliensis 12

(2): 113.1874.

Angostura tapajozensis (Ducke) B.W.P. de Albuquerque, comb. nov.

Cusparia tapajozensis Ducke, Archiv. Jard. Bot. Rio de Janeiro, 4: 100. 1925.

Angostura toxicaria (Engler) B.W.P. de Albuquerque, comb. nov.

Cusparia toxicaria Engler, in Martius, Flora Brasiliensis 12 (2): 114, tab. 24, fig. 1. 1874.

Galipee toxicaria Spruce, I. c., nom. nud.

Angosiura trombetensis (Ducke) B.W.P. de Albuquerque, comb. nov.
Cusparia trombetensis Ducke, Archiv. Jard. Bot. Rio de Janeiro, 3: 183. 1922.

Angostura ucayalina (Huber) B.W.P. de Albuquerque, comb. nov.

Cusparia ucayalina Huber, Bol. Mus. Para. Goeldi, 4: 573, fig. 4. 1906.

Angostura undulaia (Hemsley) B.W.P. de Albuquerque, comb. nov.

Cusparia undulata Hemsley, Gard. Chron. 2: 396. 1892.

\section{SUMMARY}

New cobinations for the genus Angostura Roemer \& Schultes (Rutaceae) are proposed.

(Aceito para publicação em $02 / 02 / 81$ ) 